\title{
Localization of Unbounded Operators on Guichardet Spaces
}

\author{
Jihong Zhang, Caishi Wang, Lina Tian \\ School of Mathematics, Lanzhou City University, Lanzhou, Gansu, China \\ Email: zhjhzhangjihong@163.com
}

Received 3 March 2015; accepted 23 June 2015; published 30 June 2015

\section{Abstract}

As stochastic gradient and Skorohod integral operators, $(\nabla, \delta)$ is an adjoint pair of unbounded operators on Guichardet Spaces. In this paper, we define an adjoint pair of operator $\left(\ell_{s}, \ell_{s}^{*}\right)$, where $\ell_{s}=\nabla_{s} E_{s}[C]$ with $E_{s}[C]$ being the conditional expectation operator. We show that $\ell_{s}$ (resp. $\ell_{s}^{*}$ ) is essentially a kind of localization of the stochastic gradient operators (resp. Skorohod integral operators $\delta$ ). We examine that $\ell_{s}$ and $\ell_{s}^{*}$ satisfy a local CAR (canonical ani-communication relation) and $\left(\ell_{s}^{*}\right)_{s \geq 0}$ forms a mutually orthogonal operator sequence although each $\ell_{s}$ is not a projection operator. We find that $\ell_{s}$ is s-adapted operator if and only if $\nabla_{s}$ is s-adapted operator. Finally we show application exponential vector formulation of $Q S$ calculus.

\section{Keywords}

Stochastic Gradient Operator, Skorohod Integral Operator, Localization, Ex-Ponential Vector, Guichardet Spaces

\section{Introduction}

The quantum stochastic calculus [4] [6] developed by Hudson and Parthasarathy is essentially a noncommutative extension of classical Ito stochastic calculus. In this theory, annihilation, creation, and number operator processes in boson Fock space play the role of "quantum noises", [2] which are in continuous time. On the other hand, the quantum stochastic calculus has been extended by Hitsuda is by means of the Hitsuda-Skorohod integral of anticipative process [3] [9] and the related gradient operator of Malliavin calculus. In this noncausal formulation the action of each QS integral is defined explicitly on Fock space vectors, and the essential quantum Ito formula is seen in terms of the Skorohod isometry.

In 2002, Attal [1] unify and extend both of the above approaches on Guichardet spaces. In this note, explicitly definitions of QS integrals provided and introduced no unnatural domain limitations. Moreover, maximality of operator domains is demon-strated for these QS integrals on Guichardet spaces.

In this argument, we define an adjoint pair of operator $\left(\ell_{s}, \ell_{s}^{*}\right)$, where $\ell_{s}=\nabla_{s} E_{s}[C]$ with $E_{s}[C]$ being 
the conditional expectation (operator). The motivation for this study comes from the following observations. It is known that $E_{s}[C]$ is a projection operator on Guichardet Spaces. Hence, restricted to the range of $E_{s}[C], \ell_{s}$ coincides with the stochastic gradient operator $\nabla_{s}$. We show that $\ell_{s}$ (resp. $\ell_{s}^{*}$ ) is essentially a kind of localization of the stochastic gradient operators (resp. Skorohod integral operators $\delta$ ). We examine that $\ell_{s}$ and $\ell_{s}^{*}$ can be called a local stochastic gradient operators (resp. local Skorohod integral operators $\delta$ ). Then, it is necessary and important to study a pair of operator $\left(\ell_{s}, \ell_{s}^{*}\right)$.

This paper is organized as follows. In Section 2, we fix some necessary notations and recall main notions and facts about unbounded operators on Guichardet spaces. In Section 3, Section 4 and Section 5, we state our main results. We first examined that $\ell_{s}$ and $\ell_{s}^{*}$ satisfy a local CAR (canonical anti-communication relation) and $\left(\ell_{s}^{*}\right)_{s \geq 0}$ forms a mutually orthogonal operator sequence although each's is not a projection operator. We find that $\ell_{s}$ is s-adapted operator if and only if $\nabla_{s}$ is s-adapted operator. Finally we show application exponential vector formulation of QS calculus.

\section{Unbounded Operators on Guichardet Spaces}

In this section, we fix some necessary notations and recall main notions and facts about unbounded operators on Guichardet spaces. For detail formulation of unbounded operators, we refer reader to [1].

Let $\mathbb{R}_{+}$be the set of all nonnegative real numbers and $\Gamma$ the finite power set of $\mathbb{R}_{+}$, namely

$$
\Gamma \doteq\left\{\sigma \mid \sigma \subset \mathbb{R}_{+}, \# \sigma<\infty\right\}
$$

where $\# \sigma$ denotes the cardinality of $\sigma$ as a set, with $\Gamma^{(n)}$ denoting the collection of $n$ element subsets. Obviously, $\Gamma=\bigcup_{n>0} \Gamma^{(n)}$. Particularly, let $\varnothing \in \Gamma^{(0)}$ be an atom of measure 1 . We denote by $L^{2}(\Gamma)$ the usual space of square integral real-valued functions on $\Gamma$.

Fixing a complex separable Hilbert space $\eta$, Guichardet space tensor product $\eta \otimes L^{2}(\Gamma)$, which we identify with the space of square-integrable functions $L^{2}(\Gamma ; \eta)$, and is denoted by $F$. Guichardet space enjoys a continuous tensor product structure: for each $s \geq 0$ the map

$$
f \otimes g(\omega)=f\left(\omega_{s)}\right) g\left(\omega_{(s}\right)
$$

where $\omega_{s)}=\omega \bigcap[0, s), \omega_{(s}=\omega \bigcap(s, \infty)$.

For a Hilbert space-valued map $x: \Gamma \times \mathbb{R}_{+} \rightarrow \eta$, let $\delta(x)$ be the map $\Gamma \rightarrow \eta$ given by

$$
\delta(x)(\sigma)=\sum_{s \in \sigma} x_{s}(\sigma \backslash s)
$$

when $\delta(x) \in F$, we call $x$ is Skorohod integrable, $\delta(x)$ is Skorohod integral operator on $F$ and

$$
\operatorname{Dom} \delta \doteq\left\{x \in L^{2}\left(\Gamma \times \mathbb{R}_{+}, \eta\right): \delta(x) \in F\right\}
$$

For a map $f: \Gamma \rightarrow \eta$, let $\nabla f$ and $D f$ be the maps $\Gamma \times \mathbb{R}_{+} \rightarrow \eta$ given by

$$
\nabla f(\omega, s)=f(\omega \bigcup s), \quad D f(\omega, s)=1_{\{\omega<s\}} f(\omega \bigcup s)
$$

when $f \in F$, we call $\nabla f$ and $D f$ the stochastic gradient of $f$ and the adapted gradient of $f$, respectively. Moreover,

$$
\begin{aligned}
& D o m \nabla \doteq\left\{f \in F: \nabla f \in L^{2}\left(\Gamma \times \mathbb{R}_{+}, \eta\right)\right\} \\
& D o m D \doteq\left\{f \in F: D f \in L^{2}\left(\Gamma_{s} \times \mathbb{R}_{+}, \eta\right)\right\}
\end{aligned}
$$

where $\Gamma_{s}=\{\omega \in \Gamma: \omega \subset[0, s)\}$. Obviously, if $f \in F_{s}, \nabla f=D f$, where $F_{s}=L^{2}\left(\Gamma_{s}, \eta\right)$.

Let $\Gamma_{a d}=\left\{(\omega, s) \in \Gamma \times \mathbb{R}_{+}: \omega<s\right\}$, the adapted projection on $L^{2}\left(\Gamma \times \mathbb{R}_{+}, \eta\right)$ is the orthogonal projection onto the closed subspace $L^{2}\left(\Gamma_{a d} \times \mathbb{R}_{+}, \eta\right)$ :

$$
P_{a d}:(\omega, s) \mapsto 1_{\{\omega<s\}} x_{s}(\omega)
$$

Remark 2.1 As Hilbert space operators $\delta, \nabla$ and $D$ are unbounded operators. $(\delta, D o m \delta)$ and $(\nabla, \operatorname{Dom} \nabla)$ are closed, densely defined operators. Especially, $\delta$ is adjoint operator of $\nabla$ and 


$$
\operatorname{Dom} \delta \supset \operatorname{Dom} \sqrt{N \otimes I} ; \operatorname{Dom} \nabla=\operatorname{Dom} \sqrt{N}
$$

where $N$ is the number operator, $N f(\sigma)=\# \sigma f(\sigma)$ with maximal domain and $I$ is identical operator.

Lemma 2.1 [1] Let $f \in F$ and $x: \Gamma \times \mathbb{R}_{+} \rightarrow \eta$ be Skorohod integrable, if the map

$$
(\omega, s) \mapsto\left\langle x_{s}(\omega), f(\omega \cup s)\right\rangle
$$

is integrable, then

$$
\langle\delta(x), f\rangle=\iint\left\langle x_{s}(\omega), \nabla_{s} f(\omega)\right\rangle d \omega d s .
$$

Lemma 2.2 [1] Let $x: \Gamma \times \mathbb{R}_{+} \rightarrow \eta$ be measurable. If $P_{t} x_{t} \in F$ for almost every $t \geq 0$, then

$$
D_{t} \delta(x)=\delta_{0}^{t}\left(\nabla_{t} x_{\text {. }}\right)+P_{t} x_{t},
$$

where (1) may call the canonical-commutation relations.

\section{Local Skorohod Integral and Stochastic Gradient Operators}

In the present section we state and prove our main results. We first make some preparations.

Let $C$ be an operator on $F$ with domain $V$, we define an conditioned expectation operator $E_{s}[C]$ on $F$ by the a.e. prescription

$$
\left(E_{s}[C] f\right)(\omega)=\left(C P_{s} D_{\omega_{(s}} f\right)\left(\omega_{s)}\right),
$$

with domain

$$
\left\{f \in \mathrm{D}_{s}[V]: \sigma \mapsto 1_{\{\sigma>s\}} P_{s} C P_{s} D_{\sigma} f \text { is square integral } \Gamma \rightarrow F\right\}
$$

where $\mathrm{D}_{s}[V] \doteq\left\{f \in F: P_{s} D_{\sigma} f \in V(\subset F)\right.$ for a.a. $\left.\sigma>s\right\}, \omega_{s)}=\omega \bigcap[0, s), \omega_{(s}=\omega \bigcap(s, \infty)$.

Clearly, $\mathrm{D}_{s}[V]$ is a subspce of $F$, and for any $f \in \mathrm{D}_{s}[V]$, we have $P_{s} f \in \mathrm{D}_{s}[V], D_{t} f \in \mathrm{D}_{s}[V]$ for a.a. $t>s$. Thus $\mathrm{D}_{s}[V]$ is an s-adapted subspace.

Remark 3.1 If $C$ is s-adapted(i.e. for all $f \in \operatorname{Dom} C, P_{s} f=P_{s} C P_{s} f, D_{t} C f=C D_{t} f$ for a.a. $t>s$ ), then the subspaces $F_{s} \cap D o m C$ and $F_{s} \cap D o m E_{s}[C]$ coincide, and $E_{s}[C] g=C g$ for $g$ in this subspace, it follows that

$$
E_{s}[C] P_{s} f=C P_{s} f, E_{s}[C] D_{s} f=C D_{s} f
$$

Whenever $P_{s} f$ belongs to DomC. If $C$ is densely defined, s-adapted and $E_{s}[C]$ is closable, then $E_{s}\left[C^{*}\right]=E_{s}[C]^{*}$.

Remark 3.2 $E_{s}[C]$ is s-adapted operator and $E_{s}[C] \in F$.

Definition 3.1 For $s \in \mathbb{R}_{+}$, we call $\ell_{s}=\nabla_{s} E_{s}[C]$ the local stochastic gradient operator and its adjoint operator $\ell_{s}^{*}=\delta E_{s}[C]$ is the local Skorohod integral operator. And operator domain of $\ell_{s}$ is given by

$$
\operatorname{Dom}_{s} \doteq \operatorname{Dom}_{s} \cap \operatorname{DomE}_{s}[C]
$$

where $C$ is operator on $F$.

We note that for $f \in D_{\text {Dom }}$,

$$
\begin{aligned}
& \left(\nabla_{s} E_{s}[C] f\right)(\omega)=\nabla_{s}\left(E_{s}[C] f(\omega)\right)=C P_{s} D_{\omega_{(s}} f\left(\omega_{s)} \cup s\right) \\
& \left(E_{s}[C] \nabla_{s} f\right)(\omega)=E_{s}[C]\left(\nabla_{s} f(\omega)\right)=C P_{s} D_{\omega_{(s}} f\left(\omega_{s)} \cup s\right)
\end{aligned}
$$

hence, $\nabla_{s} E_{s}[C]=E_{s}[C] \nabla_{s}$. Especially, when $C=P_{s}, f(\omega)=P_{s} P_{s} D_{\omega_{(S}} f\left(\omega_{s)}\right)$, we have $\ell_{s}=\nabla_{s}$.

Theorem 3.1 By lemma2.2, we can get the following relations

$$
\ell_{s} \ell_{s}^{*}=\ell_{s}^{*} \ell_{s}+E_{s}[C]
$$

which we may call the local CAR(canonical anti-commutation relations). 
Proof we note that

$$
\begin{aligned}
\ell_{s} \ell_{s}^{*} & =\nabla_{s} E_{s}[C] \delta E_{s}[C]=E_{s}[C] \nabla_{s} \delta E_{s}[C] \\
& =E_{s}[C]\left(\delta\left(\nabla_{s} E_{s}[C]\right)+E_{s}[C]\right)=\ell_{s}^{*} \ell_{s}+E_{s}[C]
\end{aligned}
$$

The next theorem shows that $\ell_{s}$ is not a projection operator on $F$.

Theorem $3.2 \ell_{t} \ell_{s}=0$, whenever $t \geq s$ and $t, s>0$.

Proof Let $t, s>0$ with $t \geq s$. The following algebraic relations are evident for $t \geq s$,

$$
\begin{gathered}
P_{s} P_{t} f=P_{t} P_{s} f=P_{s} f, \\
D_{t} D_{s} f=D_{t} P_{s} f=0, \\
D_{s} P_{t} f=P_{t} D_{s} f=D_{s} f .
\end{gathered}
$$

We show that $E_{t}[C] \nabla_{s} E_{s}[C]=\nabla_{s} E_{s}[C]$, thus

$$
\ell_{t} \ell_{s}=\nabla_{t} E_{t}[C] \nabla_{s} E_{s}[C]=\nabla_{t}\left(E_{t}[C] \nabla_{s} E_{s}[C]\right)=0 .
$$

We note that $\nabla_{s} \nabla_{t}^{*} \neq 0$ for $t, s>0$ with $t \neq s$, which means that the $\left(\nabla_{s}^{*}\right)_{s \geq 0}$ is not mutually orthogonal. However, the theorem below shows that the local operator sequence $\left(\ell_{s}^{*}\right)_{s \geq 0}$ is mutually orthogonal.

Theorem $3.3 \ell_{t} \ell_{s}^{*}=0$, whenever $t \neq s$ and $t, s \geq 0$.

Proof Let $t \neq s$ and $t, s \geq 0$. If $t>s$, then we can show that $\nabla_{t} E_{s}[C]=0$, from which it follows that

$$
\ell_{t} \ell_{s}^{*}=\nabla_{t} E_{t}[C] E_{s}[C] \delta=\left(\nabla E_{s}[C]\right) \delta=0 .
$$

Now, if $t<s$, then by the result of the first step we have

$$
\ell_{t} \ell_{s}^{*}=\left(\ell_{s} \ell_{t}^{*}\right)^{*}=0
$$

This completes the proof.

Theorem $3.4 \ell_{s}$ is s-adapted operator if and only if $\nabla_{s}$ is s-adapted operator.

Proof we know that $\ell_{s}=\nabla_{s} E_{s}[C]$ and $E_{s}[C]$ is s-adapted operator. we have

$$
P_{s} \ell_{s} f=P_{s} \nabla_{s} E_{s}[C] f,
$$

$D_{t} \ell_{s} f=D_{t} \nabla_{s} E_{s}[C] f$, for a.a. $t \geq s$, obviously, if $\nabla_{s}$ is s-adapted, then $P_{s} \nabla_{s} E_{s}[C] f=\nabla_{s} E_{s}[C] P_{s} f$ and $D_{t} \nabla_{s} E_{s}[C] f=\nabla_{s} E_{s}[C] D_{t} f$ for a.a. $t \geq s$. On the other hand, if $\ell_{s}$ is s-adapted, $\nabla_{s}$ is also s-adapted.

\section{Application to Exponential Vector Formulation of QS Calculus}

Recall that in the exponential vector formulation of QS calculus, all processes are defined on a domain of the algebraic tensor product form $V_{0} \odot \mathcal{E}(S)$, where $V_{0}$ is a dense subspace of $\eta$ and $\mathscr{E}(S) \doteq \operatorname{Lin}\{\varepsilon(\varphi): \varphi \in S\}$ which $S$ is a subset of $L^{2}\left(\mathbb{R}_{+}\right)$and $\varepsilon(\varphi)$ denotes the expential vector of the test function $\varphi$ which in Guichardet spaces given by $\varepsilon(\varphi)=\prod_{s \in \sigma} \varphi(s)$.

For all $s$ and a.a. $t$, we have

$$
P_{s} v \varepsilon(\varphi)=v \varepsilon\left(\varphi_{[0, s)}\right) \text {, and } D_{t} v \varepsilon(\varphi)=\varphi(t) v \varepsilon\left(\varphi_{[0, t)}\right),
$$

since, the domain of the form $V_{0} \odot \mathcal{E}(S)$ are s-adapted. Note the a.e. identity

$$
D_{\tau} v \varepsilon(\varphi)=v \varepsilon\left(\varphi_{[0, t)}\right) \varepsilon\left(\varphi_{[t, \infty)}\right)(\tau) \text {, where } t=\wedge \tau \text {. }
$$

Theorem $4.1 \ell_{s}$ be an operator on $F$ with domain of the form $V_{0} \odot \varepsilon(S)$. Then $\ell_{s}$ is s-adapted if and only if, for all $v \in V_{0}$ and $\varphi \in S$ :

$$
\ell_{s} v \varepsilon\left(\varphi_{[0, s)}\right) \in F_{s} ; \quad \ell_{s} v \varepsilon(\varphi)=\ell_{s} v \varepsilon\left(\varphi_{[0, s)}\right) \otimes \varepsilon\left(\varphi_{[s, \infty)}\right),
$$

where $F_{s}=\eta \otimes L^{2}\left(\Gamma_{s}\right)$.

Proof By definition of $\ell_{s}, \ell_{s}$ be an operator on $F$ with domain of the form $V_{0} \odot \varepsilon(S)$. We note that if $\ell_{s}$ is s-adapted, then $\ell_{s} v \varepsilon\left(\varphi_{[0, s)}\right) \in F_{s}$. Let $v \in V_{0}$ and $\varphi \in S$, by (4), for a.a. $\omega>s$, 


$$
P_{s} D_{\omega} v \varepsilon(\varphi)=v \varepsilon\left(\varphi_{[0, s)}\right) \varepsilon\left(\varphi_{[s, \infty)}\right)(\omega)
$$

and so, for a.a. $\omega$,

$$
\begin{aligned}
\left(\ell_{s} v \varepsilon(\varphi)\right)(\omega) & =\ell_{s} v \varepsilon\left(\varphi_{[0, s)}\right)\left(\omega_{s)}\right) \otimes \varepsilon\left(\varphi_{[s, \infty)}\right)\left(\omega_{(s}\right) \\
& =\left(\ell_{s} v \varepsilon\left(\varphi_{[0, s)}\right)\right) \otimes\left(\varepsilon\left(\varphi_{[s, \infty)}\right)\right)(\omega) .
\end{aligned}
$$

\section{Acknowledgements}

The authors are extremely grateful to the referees for their valuable comments and suggestions on improvement of the first version of the present paper. The authors are supported by National Natural Science Foundation of China (Grant No. 11261027 and No. 11461061).

\section{References}

[1] Attal, S. and Lindsay, J.M. (2004) Quantum Stochastic Calculus with Maximal Operator Domains. The Annals of Probability, 32, 488-529. http://dx.doi.org/10.1214/aop/1078415843

[2] Wang, C.S., Lu, Y.C. and Chai, H.F. (2011) An Alternative Approach to Privault's Discrete-Time Chaotic Calculus. J.Math.Anal.Appl., 373, 643-654. http://dx.doi.org/10.1016/j.jmaa.2010.08.021

[3] Hitsuda, M. (1972) Formula for Brownian Partial Derivatives. Proceedings of the 2nd Japan-USSR Symposium on Probability Theory Commun.Math.Phys., 2, 111-114.

[4] Hudson, R.L. and Parthasarathy, K.R. (1984) Quantum Ito’s Formula and Stochastic Evolutions. Commun.Math.Phys., 93, 301-323. http://dx.doi.org/10.1007/BF01258530

[5] Kuo, H.H. (1996) White Noise Distribution Theory. Probability and Stochastics Series, CRC Press.

[6] Meyer, P.A. (1993) Quantum Probability for Probabilists. Lecture Notes in Mathematics, Spring-Verlag, Berlin. http://dx.doi.org/10.1007/978-3-662-21558-6

[7] Privault, N. (2009) Moment Identities for Skorohod Integrals on the Wiener Space and Applications. Electronic Communications in Probability, 14, 116-121.

[8] Privault, N. (2010) Random Hermite Polynomials and Girsanov Identities on the Wiener Space. Infinite Dimensional Analysis, 13, 663-675.

[9] Skorohod, A.V. (1975) On a Generalization of a Stochastic Integral. Theory Probab. Appl., 20, 219-233. 